\title{
Potential cellular signatures of viral infections in human hematopoietic cells
}

\author{
J. Mikovits ${ }^{\mathrm{a}, *}$, F. Ruscetti ${ }^{\mathrm{b}}$, W. Zhu ${ }^{\mathrm{a}}$, R. Bagnic, \\ D. Dorjsuren ${ }^{\mathrm{a}}$ and R. Shoemaker ${ }^{\mathrm{d}}$ \\ a Laboratory of Antiviral Drug Mechanisms, \\ Frederick, MD, USA \\ ${ }^{\mathrm{b}}$ Laboratory of Leukocyte Biology, DBS, National \\ Cancer Institute-Frederick Cancer Research Center, \\ Frederick, MD 21702-1201, USA \\ ${ }^{\mathrm{c}}$ Intramural Research Support Program SAIC \\ Frederick, Frederick, MD, USA \\ ${ }^{\mathrm{d}}$ Screening Technologies Branch, Developmental \\ Therapeutics Program, DCTD, Frederick, MD, USA
}

\begin{abstract}
Expression profiling of cellular genes was performed using a 10,000 cDNA human gene array in order to identify expression changes following chronic infection of human hematopoietic cells with Kapsosi's Sarcoma -associated Virus (KSHV) also known as Human Herpesvirus 8 (HHV8) and Human T cell leukemia virus-1 (HTLV-1). We performed cell-free in vitro infection of primary bone marrow derived CD34+ cells using semi-purified HHV8 and a mature IL-2 dependent T cell line, KIT 225, using highly concentrated viral stocks prepared from an infectious molecular clone of HTLV-1. Thirty days post infection, mRNA was isolated from infected cultures and uninfected controls and submitted for microarray analysis. More than 400 genes were differentially expressed more than two-fold following HHV8 infection of primary bone marrow derived CD34+ cells. Of these 400, interferon regulatory factor 4 (IRF4), cyclin B2, TBP-associated factor, eukaryotic elongation factor and pim 2 were up-regulated more than 3.5 fold. In contrast, less than 100 genes were differentially expressed more than twofold following chronic infection of a mature $\mathrm{T}$ cell line with HTLV-1. Of these, only cdc7 was up-regulated more than 3.5 fold. These data may provide insight into cellular signatures of infection useful for diagnosis of infection as well as potential targets for therapeutic intervention.
\end{abstract}

*Address for correspondence: SAIC Frederick, NCI-FCRDC, PO Box B, Bldg 439, Frederick, MD 21702-1201, USA. Tel.: +1 301 846 5610; Fax: +1 301846 6846; E-mail: Mikovits@ncifcrf.gov.

\section{Introduction}

DNA microarray technologies used for gene expression profiles of biological systems are proving to be powerful tools in identifying signatures of underlying biological conditions [26], providing a transcriptional snapshot of the molecular phenotype of a cell. Microarray technology has advanced to a level of sophistication in which expression differences can be detected revealing detailed cellular responses to diverse stimuli such as environmental, pharmacological agents or pathogens. Microarray analysis may provide signatures for pathogens that are diagnostic even when the infectious agent is unknown or cannot be isolated. To obtain signatures for specific pathogens, it is necessary to capture their effects on host cell gene expression. In this regard, we performed in vitro infections of target cell populations using two viruses, HHV8 and HTLV1 [5,18]. HHV8, a $\gamma 2$ herpesvirus, and HTLV-1, a retrovirus, are both poorly infectious and are associated with tumors of hematopoietic cells. In addition, infections with both of these viruses are characterized by a long latency period prior to onset of pathogenesis and disease occurs in only a small subset of infected individuals.

To date, few studies have been published using gene arrays to detect genome-wide changes in gene expression in response to pathogens $[9,14,21,27]$. This likely reflects the fact that the technology has only recently become generally available. Difficulties with respect to obtaining patient material and identification of infected cells in vivo also complicate these types of analyses. However, the use of highly purified populations of hematopoietic cells isolated using specific cell surface markers and immuno-magnetic separation or high speed cell sorters provide an opportunity to perform in vitro infections in controlled experiments and easily generate the $0.2-2 \mu \mathrm{g}$ of polyA + RNA required for microarray analysis.

In these studies we describe microarray analysis using the Incyte UniGemV 10, 000 gene array of HHV8 and HTLV-1 infected hematopoietic target cells and 
Table 1

mRNAs that increase $>3$ fold in productively HHV8 infected CD34+ hematopoietic progenitor cells

\begin{tabular}{llr}
\hline Gene bank accession \# & Name of gene & Fold increase \\
\hline U52682 & Interferon Regulatory Factor 4 (IRF) & 13.4 \\
AL04861 & ribosomal protein L4 & 5.6 \\
AJ000480 & Phosphoprotein reg by mitogenic pathways & 5.5 \\
AJ929696 & ribosomal protein PO & 4.4 \\
AI192628 & ribonuclease 6 precursor & 4.0 \\
A1826416 & eukaryotic translation elongation factor 2 & 4.0 \\
X97999 & TBP-associated factor & 3.9 \\
AI634033 & human pim 2 & 3.7 \\
N87720 & cyclin B2 & 3.7 \\
M34664 & Heat shock 60 kD protein & 3.6 \\
AA313584 & eukaryotic translation initiation factor 3 & 3.5 \\
U37122 & adducin & 3.5 \\
L13923 & fibrillin 1 & 3.4 \\
U10691 & melanoma antigen & 3.4 \\
AF004162 & n-myc & 3.2 \\
U04898 & RAR-related orphan receptor A & 3.1 \\
X06431 & CD48 (B cell membrane protein) & 3.0 \\
U87967 & CD 39 antigen & 3.0 \\
\hline
\end{tabular}

suggest that data obtained using analyses such as these can be used to as signatures of infectious agents and identify molecular targets for therapeutic intervention.

\section{Materials and methods}

\subsection{Cell isolation and culture}

The KIT225 cell line [12] was cultured in RPMI 1640 media containing $10 \%$ FBS, penicillin, streptomycin, L-glutamine (Gibco-BRL, Gaithersburg, MD) containing 50 units/ml recombinant IL2. Primary stem cell CD34+ progenitors were isolated from human umbilical cord blood or adult bone marrow by ficoll hypaque density centrifugation and isolation of subpopulations done using antibody coupled microbeads and magnetic cell separation (Miltenyi Biotec, Auburn CA). Hematopoietic progenitor cells were cultured in the following media: Iscove's Modified Dulbecco's medium containing $50 \mathrm{ng} / \mathrm{ml}$ recombinant human (rh) thrombopoietin, $50 \mathrm{ng} / \mathrm{ml}$ stem cell factor (rh-SCF), $50 \mathrm{ng} / \mathrm{ml}$ rh-FLT 3 ligand (Prepotech, NJ) and 20\% horse serum.

\subsection{Virus propagation}

HHV8: Concentrated stocks were prepared as previously described using BCBL-1, a latently HHV8 infected cell line containing $\sim 70$ copies HHV8/cell [20]. The viral pellet was re-suspended at $100 \mathrm{X}$ in serum free RPMI and copy number quantified by real time Taqman
PCR for ORF 22 [19]. Viral Stocks were stored in liquid nitrogen until needed for infection. Cells were infected at a multiplicity of infection (moi) of 10 (copies per cell) by exposing cells to virus for 2-4 hours in a $37^{\circ} \mathrm{C}$ shaking water bath. Following exposure, cells were washed once to remove non-adsorbed virus and cultured in the appropriate media. Cells were cultured 21-30 days (5-6 passages) and tested for expression of both latent and lytic genes representing a persistently infected culture. In this way, $>50 \%$ of the culture was demonstrated to be infected. For producing HTLV-1: 293T cells were transfected with an infectious molecular clone of HTLV-1 using the calcium phosphate method as previously described [7]. Stocks were normalized to $10 \mathrm{ng} / \mathrm{ml}$ and used to infect KIT225 at a moi of 10 .

\subsection{Sample preparation and analysis with DNA array}

Total cellular RNA was prepared using the TRIZOL reagent (Gibco, Life Technologies) according to manufacturer's instructions. Poly A+ RNA was isolated using the oligotex mRNA mini kit (Qiagen). RNA from two separate experiments was pooled to control for error due to experimental variation. Microarray analysis of Poly A + RNA was performed using the 10,000 gene Human UnigemV system (Incyte Pharmaceuticals, Human Genome Systems) where fluorescent cDNA probes, labeled with the Cy5 dye were prepared from the infected population and $\mathrm{Cy} 3$ dye probe prepared from the control (uninfected) population. The UniGEM V microarray contains Incyte se- 
Table 2

mRNAs that decrease $>3$ fold in productively HHV8 infected CD34+ hematopoietic progenitor cells

\begin{tabular}{llc}
\hline Gene bank accession \# & Name of gene & Fold decrease \\
\hline M26683 & IFN $\gamma$ treatment inducible RNA & 9.5 \\
L16510 & cathepsin B & 8.1 \\
M80815 & fucosidase, $\alpha$ L1 & 6.0 \\
M12937 & ferritin & 5.9 \\
M98398 & CD36 antigen (thrombospondin receptor) & 5.4 \\
M73720 & Carboxypeptidase A3 & 5.3 \\
X16832 & cathepsin H & 5.1 \\
A1243916 & apolipoprotein C-I & 4.9 \\
U15085 & MHC class II, DM $\beta$ & 4.8 \\
K00396 & apolipoprotein E & 4.7 \\
U78045 & MMP 12 (macrophage elastase) & 4.6 \\
J03015 & prosaposin & 4.5 \\
V000522 & MHC class II DR $\beta$ & 4.2 \\
X07743 & 4.0 \\
AI911758 & pleckstrin & 3.9 \\
M17955 & HLA-DR $\alpha$ & 3.7 \\
L20321 & MHC class II DQ $\beta$ & 3.5 \\
J03143 & serine/threonine kinase 2 & 3.4 \\
M11313 & IFN $\gamma$ receptor 1 & 3.3 \\
M25246 & $\alpha-2$ macroglobulin & 3.1 \\
M81695 & vimentin & 3.1 \\
X07819 & CD11C (p150) intgrin & 3.1 \\
Z22968 & MMP 7 (matrilysin) & 3.0 \\
\hline & macrophage associated- antigen &
\end{tabular}

quence verified cDNA clones representing unique human genes identified in NCBI's UniGene database. Incyte has verified all clones by re-sequencing. A complete list of known genes on UniGEM $\mathrm{V}$ is available from Incyte or the UniGene website: http://www.ncbi.nlm.nih.gov/UniGene/index.html.

\section{Results and discussion}

We used microarray technology to determine genes deregulated by productive infection of hematopoietic cells with HHV8 and HTLV1. RNA samples were prepared several passages following infection during productive viral replication in the majority of cells as determined by flow cytometry using an antibody to HHV8 ORF 8.1 [4]. In these studies two preparations of infected cell and control cell target RNAs were prepared from primary CD34+ hematopoietic cells derived from two different donors, controlling for the possibility that changes in RNA levels might reflect genetic differences in host cells. These cells were identified as a progenitor population that was primarily myeloid as evidenced by the surface expression of CD33 and no late lineage markers. Table 1 shows the mRNAs that increase $>3$ fold after HHV8 infection. We confirmed the 6 of the 16 mRNAs predicted to be increased $>3$ fold (IRF4, pim2, n-myc, cyclin B2 and heat shock protein, CD48 and CD39) either by northern analysis (using clones purchased from Incyte), ribonuclease protection analysis or RT-PCR (not shown). All attempts to confirm a predicted change in gene expression were successful. As shown in Tables 2 and 4, infection with either HHV8 or HTLV-1 resulted in decreased expression of genes involved in immune or inflammatory responses. These down-regulated genes may indicate pathways used by the virus for persistence and evasion of the immune response.

HHV-8 is a human lymphotropic herpes virus implicated in the pathogenesis of Kaposi's sarcoma (KS) [5, 17] and several hematopoietic and lymphoproliferative diseases including body cavity based or primary effusion lymphoma and multicentric Castleman's disease $[3,6]$. KS itself is a hyperproliferation of poorly differentiated endothelial cells and is associated with extreme neovascularization. HHV8 has been shown to be present in $\mathrm{KS}$ spindle cells [6], endothelial cells [8], macrophages, B lymphocytes [13] and circulating CD34+ progenitor cells vivo [11]. One potentially exciting result was the 13.5 fold increase in IRF-4. Interestingly, this gene has been reported to be deregulated in chronic myeloid leukemia (CML) [22] and other mature lymphoid malignancies [25]. Further, its expression correlates with response to interferon alpha therapy in CML. KS is clinically responsive to Interferon alpha therapy. 
Table 3

mRNAs that increase $>2.5$ fold in HTLV-1 infected KIT225 T Cell Line

\begin{tabular}{llc}
\hline Gene bank accession \# & Name of gene & Fold increase \\
\hline AF015592 & CDC7 & 9.2 \\
AB011173 & KIAA0601 protein & 3.4 \\
NM_001565 & small inducible cytokine subfamily B, 10 & 2.7 \\
M55265 & casein kinase 2 & 2.6 \\
U58196 & interleikin enhancer binding factor I & 2.5 \\
NM_002156 & heat shock 60 kD protein 1 & 2.5 \\
\hline
\end{tabular}

Table 4

mRNAs that decrease $>3$ fold in HTLV-1 infected KIT225 T Cell Line

\begin{tabular}{llr}
\hline Gene bank accession \# & Name of gene & Fold decrease \\
\hline M18737 & granzyme A & 12.7 \\
M_000885 & Integrin, $\alpha$-4 (CD49D) & 8.7 \\
NM_000700 & annexin A1 & 8.5 \\
A1271688 & cyclin G2 & 5.1 \\
X00437 & T-cell receptor $\beta$ cluster & 4.9 \\
U25804 & caspase 4 & 4.7 \\
S82807 & Thyrotropin receptor & 4.4 \\
V00522 & MHC class II DR $\beta 5$ & 4.1 \\
M32578 & MHC class II DR $\beta 1$ & 3.7 \\
NM_000072 & CD36 antigen (collagen type I receptor) & 3.4 \\
M17846 & MHC class II DQ $\alpha$ & 3.4 \\
NM_00531 & IFN $\gamma$-inducible protein 16 & 3.4 \\
M29204 & transcription factor 9 & 3.3 \\
M29696 & Il-7 receptor & 3.1 \\
U13697 & caspase-1 & 3.1 \\
AW385690 & fibronectin 1 & 3.0 \\
AW157187 & Tat interactive protein (60 kD) & 3.0 \\
NM_007315 & STAT 1 & 3.0 \\
\hline
\end{tabular}

HTLV1, a retrovirus virus etiologically associated with adult $\mathrm{T}$ cell leukemia [18], is oncogenic in a multi step process, initially by the deregulation of the cell cycle control of proliferation by a mechanism that is not well understood. The deregulation of cell proliferation facilitates further genetic changes allowing $\mathrm{T}$ cells to proceed to frank malignancy [23]. A systematic analysis of genes deregulated by acute HTLV-1 infection has until recently, not been possible due to the poorly infectious nature of the virus, the lack of an infectious molecular clone and a de novo infection system. We have recently described an infectious clone, which has been shown to immortalize cells following cell free infection in vitro [7] enabling us to analyze deregulated gene expression following acute infection. To better understand the molecular mechanisms allowing for IL-2 independent growth, an immortalized T cell, KIT 225, which remains IL-2 dependent, was infected with HTLV-1. The resulting IL-2 independent cell line was compared with the IL-2 dependent cell line using UniGEM V. In Table 3, genes that increased $>3.0$ fold following infection of KIT225 with HTLV1 are shown. The single gene (cdc7) predicted to be increased $>9$ fold was confirmed by northern analysis. The cdc7 gene is an extremely promising signature of chronic HTLV-1 infection for the gene product is necessary for initiation of S phase, DNA replication and in mismatch repair of DNA [16].

Only one previous study has described expression profiling of HTLV-1 infected cells [10]. However, the data presented in this study is limited by the fact that Harhaj et al. compared HTLV-1 immortalized cell lines to normal activated PBMC cells. The limitation of this type of comparison is underscored by studies, which show different activation modes result in different patterns of gene expression [24].

The significance of the viruses examined in this study lies in the indolent nature of diseases caused by these viruses. Only a small percentage of infected individuals develop frank tumors with a latency period from infection to onset of disease measured in decades. By this time numerous genetic changes have occurred in the target cell population and the tumors are quite aggressive. This study is limited by the fact that only a single microarray is performed on one host cell line or purified primary cell culture. Additional arrays performed on other target cell populations or cell lines may yield different patterns. However, in studies us- 
ing microarrays to catalog hematological malignancies from primary patient cells, as more samples are analyzed distinct patterns of gene expression for specific disease classifications emerged [1,2]. The ability to catalog cellular transcriptional profiles for both acute and persistent infection combined with the advances in the isolation of hematopoietic target cells, makes this approach particularly attractive for diagnosis and monitoring efficacy of therapy [15] in hematopoietic malignancies with viral etiologies.

\section{Acknowledgements}

We thank Ms Lisa Gray for assistance in the preparation of the manuscript. This project has been funded in whole or in part with Federal funds from the National Cancer Institute, National Institutes of Health, under contract No. N01-CO-56000. The content of this publication does not necessarily reflect the views or policies of the Department of Health and Human Services, nor does mention of trade names, commercial products or organization imply endorsement by the US Government.

\section{References}

[1] A. Alizadeh, M. Eisen, D. Botstein, P.O. Brown and L.M. Staudt, Probing lymphocyte biology by genomic-scale gene expression analysis, Journal of Clinical Immunology 18 (1998), 373-379

[2] A.A. Alizadeh, M.B. Eisen, R.E. Davis, C. Ma, I.S. Lossos, A. Rosenwald, J.C. Boldrick, H. Sabet, T. Tran, X. Yu, J.I. Powell, L. Yang, C.E. Marti, T. Moore, J. Jr. Hudson, L. Liu, D.B. Lewis, R. Tibshirani, G. Sherlock, W.C. Chan, T.C. Greiner, D.D. Weisenburger, J.O. Armitage, R. Warnke and L.M. Staudt et al., Distinct types of diffuse large B-cell lymphoma identified by gene expression profiling, Nature $\mathbf{4 0 3}$ (2000), 503-511

[3] E. Cesarman, Y. Chang, P.S. Moore, J.W. Said and D.M. Knowles, Kaposi's sarcoma-associated herpesvirus-like DNA sequences in AIDS-related body-cavity-based lymphomas, New England Journal of Medicine 332 (1995), 1186-1191.

[4] B. Chandran, C. Bloomer, S.R. Chan, L. Zhu, E. Goldstein and R. Horvat, Human herpesvirus-8 ORF K8.1 gene encodes immunogenic glycoproteins generated by sliced transcripts, Virology 249 (1998), 140-149.

[5] Y. Chang, E. Cesarman, M.S. Pessin, F. Lee, J. Culpepper, D.M. Knowles and P.S. Moore, Identification of herpesviruslike DNA sequences in AIDS-associated Kaposi's sarcoma, Science 266 (1994), 1865-1869.

[6] N. Dupin, C. Fisher, P. Kellam, S. Ariad, M. Tulliez, N. Franck, E. van Marck, D. Salmon, I. Gorin and J.-P. Escande et al., Distribution of human herpesvirus- 8 latently infected cells in Kaposi's sarcoma, multicentric Castleman's disease, and primary effusion lymphoma, Proc Natl Acad Sci USA 96 (1999), 4546-4551.
[7] D. Derse, J. Mikovits, M. Polianova, B.K. Felber and F.W. Ruscetti, Virions released from cells transfected with a molecular clone of HTLV-1 give rise to primary and secondary infections of T-cells, J. Virol 69 (1995), 1907-1912.

[8] O. Flore, S. Rafil, S. Ely, J.J. O'Leary, E.M. Hyjek and E. Cesarman, Transformation of primary human endothelial cells by Kaposi's sarcoma-associated herpesvirus, Nature 394 (1998), 588-592.

[9] G.K. Geiss, R.E. Bumgarner, M.C. An, M.B. Agy, A.B. van't Wout, E. Hammersmark, V.S. Carter, D. Upchurch, J.I. Mullins and M.G. Katze, Large-scale monitoring of host cell gene expression during HIV-1 infection using cDNA microarrays, Virology 266 (2000), 8-16.

[10] E.W. Harhaj, L. Good, G. Xiao and S.C. Sun, Gene expression profiles in HTLV-I-immortalized T cells; deregulated expression of genes involved in apoptosis regulation, Oncogene 18 (1999), 1341-1349.

[11] M. Henry, A. Uthman, A. Geusau, A. Rieger, L. Furci, A. Lazzarin, P. Lusso and E. Tschachler, Infection of Circulating CD34+ Cells by HHV-8 in Patients with Kaposi's Sarcoma, J. Invest. Dermatol. 113 (1999), 613-616.

[12] T. Hori, T. Uchiyama, M. Tsudo, H. Umadome, H. Ohno, S. Fukuhara, K. Kita and H. Uchino, Establishment of an interleukin 2-dependent human $\mathrm{T}$ cell line from a patient with T cell chronic lymphocytic leukemia who is not infected with human T cell leukemia/lymphoma virus, Blood 70 (1987), 1069-1072.

[13] S. Kliche, E. Kremmer, W. Hammerschmidt, U. Koszinowski and J. Haas, Persistent Infection of Epstein-Barr Virus-Positive B Lymphocytes by Human Herpesvirus 8, J. Virol. 72 (1998), 8143-8149.

[14] I.D. Manger and D.A. Relman, How the host 'sees' pathogens: global gene expression responses in infection, Current Opinion in Immunology 12 (2000), 215-218.

[15] M.J. Marton, J.L. DeRisi, H.A. Bennett, V.R. Iyer, M.R. Meyer, C.J. Robert, R. Stoughton, J. Burchard, D. Slade, H. Dai, D.E. Jr. Bassett, P.O. Hartwell, P.O. Brown and S.H. Friend, Drug target validation and identification of secondary drug target effects using DNA microarrays, Nature Medicine 4 (1998), 1292-1301.

[16] H. Masai, N. Sato, T. Takeda and K. Arai, CDC7 kinase complex as a molecular switch for DNA replication, Frontiers in Bioscience 4 (1999), D834-D840.

[17] P.S. Moore and Y. Chang, Detection of herpesvirus-like DNA sequences in Kaposi's sarcoma in patients with and without HIV infection, New England Journal of Medicine 332 (1995), 1181-1185.

[18] B.J. Poiesz, F.W. Ruscetti, A.F. Gazdar, P.A. Bunn, J.D. Minna and R.C. Gallo, Detection and isolation of type C retrovirus particles from fresh and cultured lymphocytes of a patient with cutaneous T-cell lymphoma, Proc Natl Acad Sci USA 77 (1980), 7415-7419.

[19] R. Renne, D. Blackborn, D. Whitby, J. Levy and D. Ganem, Limited transmission of Kaposi's sarcoma-associate herpesvirus in cultured cells, Journal of Virology 72 (1998), 51825188.

[20] R. Renne, W. Zhong, B. Herndier, M. McGrath, N. Abbey, D. Kedes and D. Ganem, Lytic growth of Kaposi's sarcomaassociated herpesvirus (human herpesvirus 8 ) in culture, Natural Medicine 3 (1996), 342-346.

[21] O. Rey, M.A. Baluda and N.H. Park, Differential gene expression in neoplastic and human papillomavirus-immortalized oral keratinocytes, Oncogene 18 (1999), 827-831. 
[22] M. Schmidt, A. Hochhaus, S.A. Konig-Merediz, C. Brendel, J. Proba, G.J. Hoppe, B. Wittig, G. Ehninger, R. Hehlmann and A. Neubauer, Expression of interferon regulatory factor 4 in chronic myeloid leukemia: correlation with response to interferon alfa therapy, J Clin Oncol 18 (2000), 3331-3338.

[23] M.R. Smith and W.C. Greene, Molecular biology of the type I human T-cell leukemia virus (HTLV-1) and adult T-cell leukemia, J Clin Invest. 87 (1991), 761-766.

[24] T.K. Teague, D. Hildeman, R.M. Kedl, T. Mitchell, W. Rees, B.C. Schaefer, J. Bender, J. Kappler and P. Marrack, Activation changes the spectrum but not the diversity of genes expressed by T cells, Proc. Natl Acad. Sci. USA 96 (1999), 12691-12696.

[25] K. Tsuboi, S. Iida, H. Inagaki, M. Kato, Y. Hayami, I. Hanamura and K. Miura et al., MUM1/IRF4 expression as a frequent event in lymphopid malignancies, Leukemia 14 (2000), 449-456.

[26] R.A. Young, Biomedical Discovery with DNA Arrays, Cell 102 (2000), 9-15.

[27] H. Zhu, J.-P. Cong, G. MamTora, T. Gingeras and T. Shenk, Cellular gene expression altered by human cyto megalo virus: global monitoring with oligonucleotide arrays, Proc. Natl Acad. Sci. USA 95 (1998), 14470-14475. 


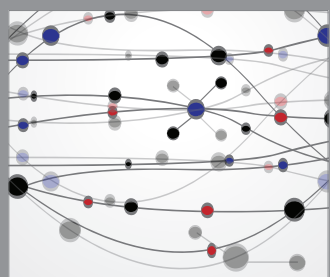

The Scientific World Journal
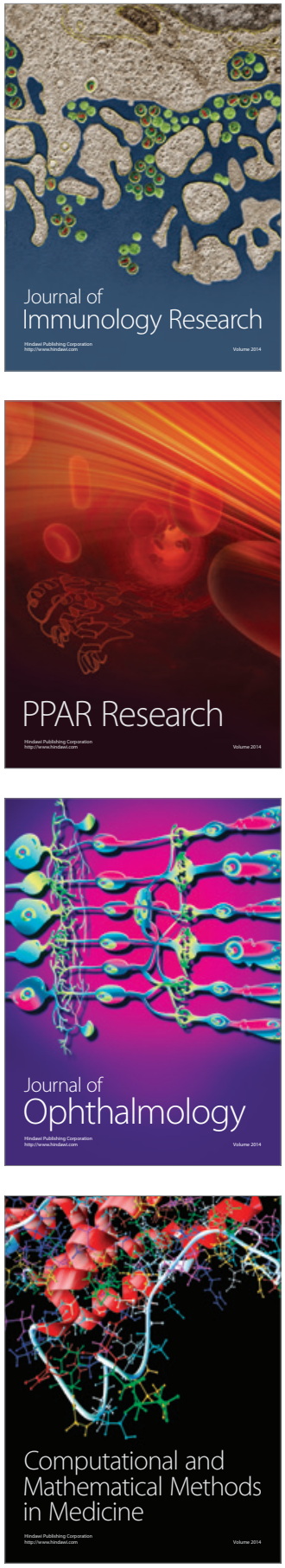

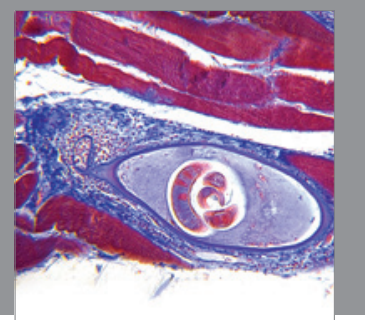

Gastroenterology

Research and Practice
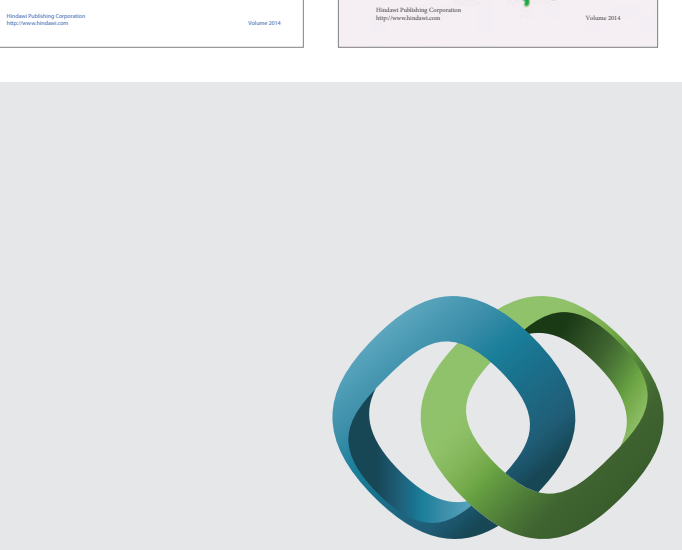

\section{Hindawi}

Submit your manuscripts at

http://www.hindawi.com
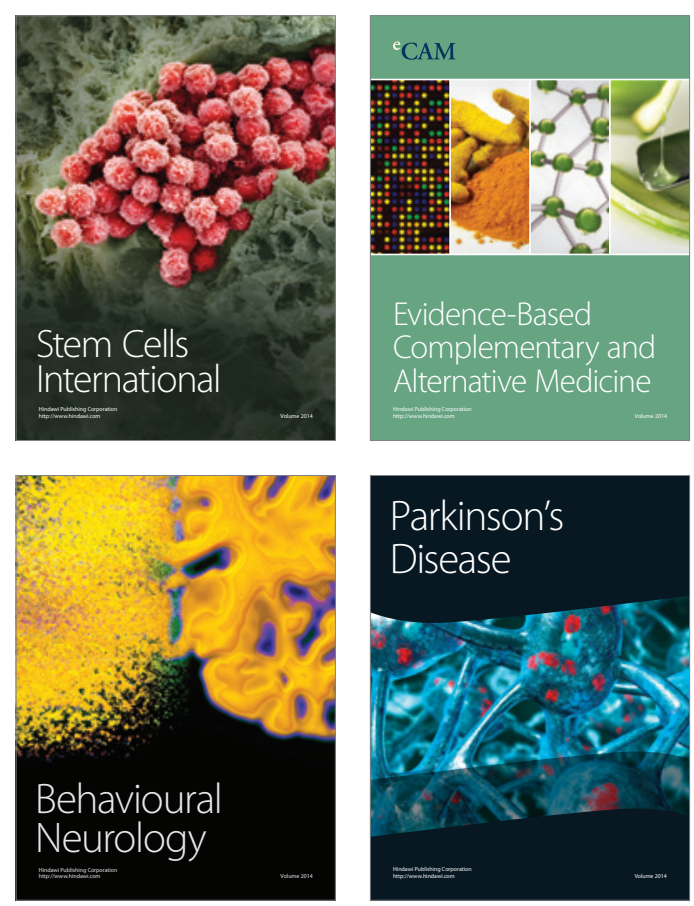

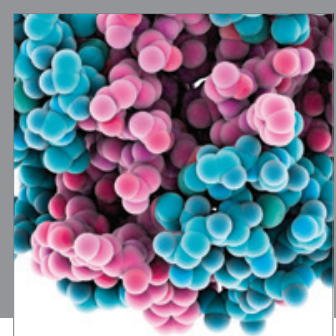

Journal of
Diabetes Research

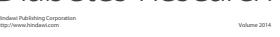

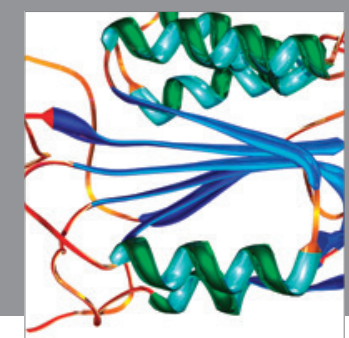

Disease Markers
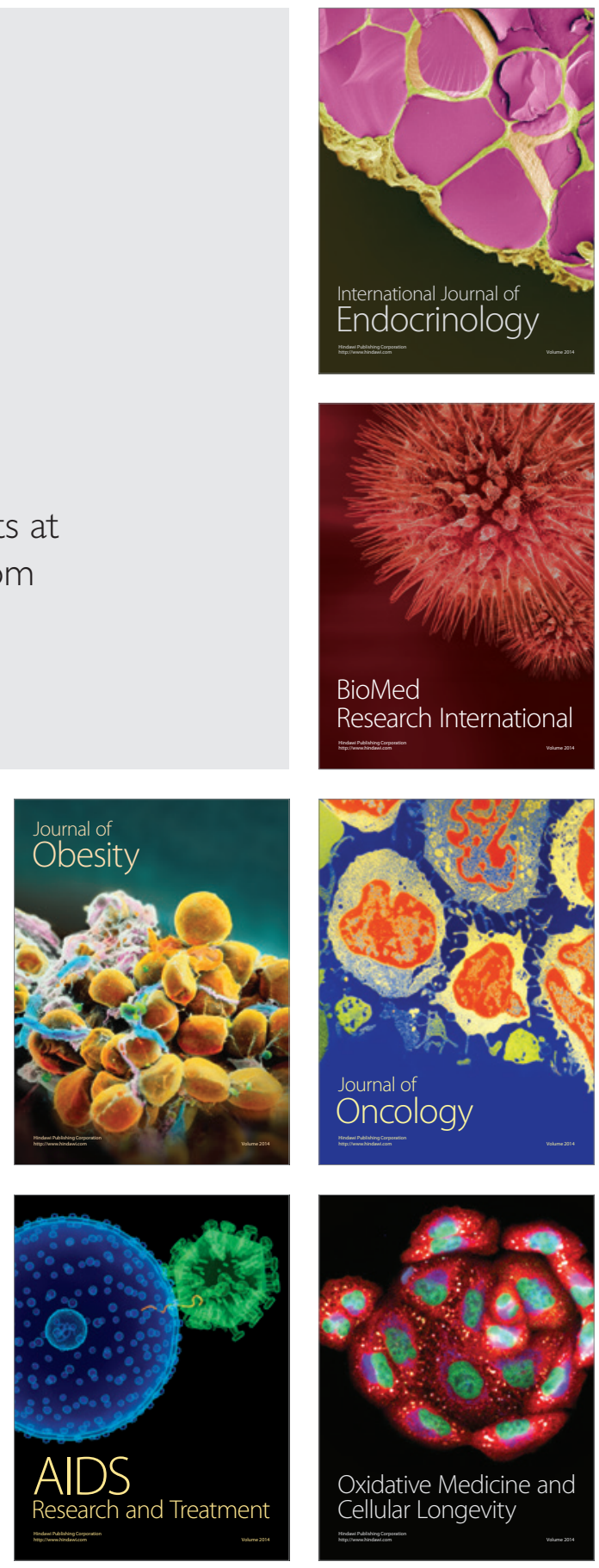